\title{
LOS FALSOS AMIGOS EN EL LÉXICO ESPAÑOL DE ORIGEN ÁRABE
}

\author{
Ziyad Muhammad Gogazeh \\ Universidad de Jordania
}

\begin{abstract}
RESUMEN
Este trabajo de investigación se centra en un estudio y análisis del léxico español de origen árabe que evolucionó semánticamente desde un punto de vista traductológico. En él se hace un estudio detallado de la influencia que ejerció la lengua árabe en el castellano a todos los niveles lingüisticos, también se recopilan todos los lexemas españoles de origen árabe que adoptaron nuevos significados en el diccionario españoly sobre los que se bace un estudio por medio de pruebas que se aplicaron a estudiantes arabo-parlantes de la asignatura Traducción del Español al Árabe del Departamento de Español en la Universidad de Jordania, para estudiar la influencia de la lengua materna del aprendiz en el proceso traductor a través de los léxicos españoles prestados del árabe que sufrieron evolución semántica y que se clasifican bajo lo que se llama en la traducción los "falsos amigos".

Palabras clave: Falsos amigos, árabe, español, léxico.

ABSTRACT

This article is based on a study and linguistic analysis of the Spanish words borrowed from the Arabic language. The meaning of these loan words has deviated, to varying degrees, from there original meanings in Arabic. Such words are often referred it as false friends, e.g. "alcalde". The study provides a detailed analysis of the influence of the Arabic language on the Spanish language at all the linguistic levels. In fact the corpus consists of all such words in Spanish as listed in the dictionary Diccionario de arabismos de la Real Academia Española. These words were included in simple sentences and given to students of Arabic - Spanish course at the University of Jordan. The native language of the subjects is Arabic. The basic objective was to examine the influence of the mother tongue on the translation.
\end{abstract}

Keywords: False friends, Arabic, Spanish, Lexicon.

Un "falso amigo" es una palabra de otro idioma que se parece (en la escritura o en la pronunciación) a una palabra en la lengua materna del hablante, pero que tiene un significado diferente. El propio término "falso amigo" fue un calco del francés "faux amis" y fue usado por primera vez por Koessler y Derocquigny en su libro Les faux 
amis ou les trabisons du vocabulaire anglais (Los falsos amigos o las traiciones del vocabulario inglés) de 1928 .

La expresión "falsos amigos" suele emplearse en los estudios de traducción para referirse a aquellas palabras que presentan cierta semejanza en dos lenguas cuyo significado es considerablemente diferente. Esa semejanza puede arrastrar al traductor a correspondencias inadecuadas 2 .

La expresión "falsos amigos" entonces suele asociarse a la ciencia de la traductología y representa uno de los problemas más considerables cuando se habla de los problemas más sobresalientes de la traducción. Me surgió la idea de escribir este articulo después de una experiencia docente en la enseñanza de la asignatura de traducción del Español al Árabe a estudiantes arabo-parlantes en la Universidad de Jordania ya que en la traducción de un texto español que incluye palabras de origen árabe el alumno arabo- parlante suele darles a esas palabras los significados semánticos existentes en el diccionario árabe sin darse cuenta de que algunas de esas palabras prestadas del árabe han desviado su significado o en algunos casos han cambiado su significado totalmente en el diccionario español.

Este problema traductológico suele suceder entre idiomas que pertenecen a la misma familia lingüística, como por ejemplo el español y el italiano o el portugués y el francés, por ser todos estos idiomas provenientes del latín. Puede suceder también entre el árabe y el hebreo por ser lenguas semíticas, pero no sucede por ejemplo entre el español y el hebreo por ser el español una lengua latina y el hebreo una lengua semítica. Pero cuando se trata de una traducción entre el árabe y el español estos dos idiomas hacen excepción a la regla y sobre todo cuando se trata de una traducción hecha a partir del español hacia el árabe pese a la diferente pertenencia lingüística, y eso se debe a la influencia que dejó el árabe en la lengua española.

Antes de plantear el problema desde el punto de vista traductológico es necesario hablar brevemente de la influencia del árabe en el español.

\section{INFLUENCIA LINGÜÍSTICA DE LA LENGUA ÁRABE EN LA LENGUA ESPAÑOLA}

Desde los comienzos de la historia de la humanidad, el Oriente Medio siempre ha dejado huellas muy sobresalientes en la cultura europea occidental, contribuyendo de una forma directa a la cultura global del planeta. En la historia antigua, las culturas de Egipto, Mesopotamia, Persia, y Anatolia influyeron en los griegos y los romanos. Los fenicios establecieron colonias comerciales a lo largo de todo el Mediterráneo. En la Edad Media, la cultura árabe e islámica ejerció una influencia profunda en Europa occidental. Este efecto fue particularmente intenso en ciertas zonas y periodos. Fue importante por ejemplo en Sicilia, que fue conquistada en el 827 y que permaneció

${ }^{1}$ Wikipedia, La enciclopedia libre, 01/05/07, Http:/es.wikipedia.org/wiki/Falsos_amigos

2 Muñiz, Carmen, "Alcance de la gramática en la traducción", Revista española de lingüística, p. 163, 1999. 
bajo dominio islámico durante tres siglos. En los Balcanes (Grecia, Albania, exYugoslavia, Bulgaria y Rumanía), que permaneció bajo el dominio otomano desde 1300 hasta 1914, y en España y Portugal, que estuvieron bajo dominio islámico desde 712 hasta 1492, casi ocho siglos. ${ }^{3}$ La influencia de la lengua árabe en la España medieval y en Portugal fue mayor y más importante en comparación con los otros países occidentales con los cuales el Islam tuvo contacto. Los préstamos lingüísticos en la lengua española son una prueba permanente de esta influencia.

La invasión de Tariq Ibn Ziyad, que entró por Gibraltar en el 711 y de Musa Ibn Nusayr, el año siguiente, supone el inicio de un dominio militar árabe sobre una gran parte de la Península Ibérica que durará casi ocho siglos. Posteriormente se inicia una lenta expansión de los reinos cristianos hacia el sur, que culminará en 1492 con la toma del reino nazarí de Granada. Durante ese período se irán tomando y repoblando los territorios arabizados en lengua, usos y costumbres ${ }^{4}$.

La conquista de los musulmanes fue rápida; en menos de ochos años conquistaron toda Hispania a excepción de una pequeña franja en el norte de la Península, donde los núcleos de resistencia dieron lugar a los reinos cristianos peninsulares, que fueron recortando progresivamente el espacio musulmán ${ }^{5}$.

España se hizo musulmana, tomó el nombre de Al-Andalus, adoptó en gran parte las costumbres, la cultura y la lengua de los musulmanes y esa influencia desempeñó un papel decisivo en su desarrollo histórico.

Las numerosas comunidades que poblaron las ricas tierras de Al-Andalus, procedentes de lejanas tierras del Yemen, Arabia, Siria, Irak, Egipto y Marruecos, vivieron largos siglos con el elemento autóctono hispano en una asociación constructiva, pasando a veces por épocas agitadas, logrando no obstante forjar esa civilización arábigo-andaluza que nos enorgullece a todos. Desde el primer momento, el idioma árabe influyó en la formación de esas comunidades. Este idioma, enriquecido en Oriente a causa de los diferentes contactos que tuvo con las viejas culturas, floreció de manera especial en Al-Andalus, portando con él los conocimientos humanos clásicos a las academias españolas medievales, interpretando de manera fiel y segura la Filosofía, la Medicina, la Astronomía, la Botánica y haciendo de Córdoba el centro difusor y propagador de esas ciencias para el resto del mundo

3 Stewart, Devin, Arabic influence on Spanish language, Literature, and culture, Emory Resourses on the Middle East, 15-02-07, $<$ http://www.mesas.emory.edu/gmesc/pdf/9_Spain_Unit_Part_1.pdf>

${ }^{4}$ Pan, José, El legado del árabe. Consejería de Educación y Ciencia en Bélgica, 15-02-07,

<http://www.sgci.mec.es/be/media/pdfs/articulos/Mosaico083.pdf>

5 Toro Lillo, Elena, La invasión árabe. Los árabes y el elemento árabe en español, Instituto Cervantes. 12-02-07,

<http://www.cervantesvirtual.com/servlet/SirveObras/01350531966682286190680/p000000 1.htm\#I_0> 
occidental. Sin duda la influencia del árabe en España ha sido radical en todas las manifestaciones de la vida social, artística, político-militar y laboral ${ }^{6}$.

Los musulmanes llegaron a la península ibérica portando una cultura superior. Esa superioridad cultural hizo que se introdujeran muchos términos nuevos inexistentes en el español medieval y se quedaran como una prueba evidente de aquella superioridad. Como dice Menéndez Pidal: "nos enseñaron (los árabes) a proteger bien la hueste con atalayas, a enviar delante de ella algaradas, a guiarla con buenos adalides, a vigilar el campamento con robdas o rondas, a dar rebato en el enemigo descuidado" 7 .

La lengua árabe siempre ha influido en las lenguas de los pueblos donde llegó el Islam y eso se debe a que el árabe está vinculado con El Quran; y siendo este libro sagrado intraducible, excepto al nivel de los significados, pues pierde su consagración cuando se vierte a otro idioma, los pueblos que se hicieron musulmanes de las tierras que cayeron bajo el dominio musulmán se encontraron ante la necesidad de aprender el árabe a fin de practicar los ritos de la religión musulmana.

La llegada de los musulmanes a España viene acompañada con una lengua de naturaleza diferente en sus manifestaciones escritas y orales de las lenguas románicas. Esta lengua - el árabe - fue impuesta como la lengua formal y de cultura junto al latín, que era una lengua coloquial y fragmentada y carecía de normalización. Mucha gente dominaba ambas lenguas convirtiendo Al-Andalus en una sociedad bilingüe hasta el siglo XI o XII.

La influencia del árabe ha sido muy sobresaliente casi a todos los niveles lingüísticos y sobre todo a nivel léxico. A nivel fonológico Cano Aguilar en su libro El Español a través de los tiempos afirma que el español no tomó prestado ningún fenómeno lingǘstico del árabe: "Todos los estudios realizados sobre correspondencia de fonemas de una lengua a la otra han resultado negativos: los respectivos sistemas fonológicos fueron siempre impermeables el uno al otro ${ }^{8}$.

Pero Cano Aguilar vuelve a decir que el efecto de los prestamos lingüísticos del árabe rectifica la frecuencia de ciertos tipos de acentuación: se incrementaron las palabras oxítonas y proparoxítonas y se produjo el aumento de polisílabas: almogávar. Berenjena. ${ }^{9}$

A nivel morfológico el árabe influyó en el Español en ciertos aspectos como la anteposición del artículo "al-" a numerosos sustantivos, a diferencia del francés o el catalán (albornoz / bornus; algodón / coton. La fusión de ese artículo con el lexema del sustantivo permite el uso de artículos romances: la almohada, el alcalde.....en

\footnotetext{
${ }^{6}$ Mekinnasi, A., Léxico de las palabras españolas de origen árabe, Madrid, Cremades, 1965, p.3.

${ }^{7}$ Menéndez Pidal, Manual de Gramática Histórica Española, Madrid, Ed. Espasa Calpe. 1993, p.32.

${ }^{8}$ Cano Aguilar, R. El español a través de los tiempos, 1999, p.52.

${ }^{9}$ Idem, p.145.
} 
muchos casos, las palabras se han formado con el artículo "al-" unido a la base latina $\left(\right.$ almeja < ár. $a l+$ lat. mitulu, alpiste $(<\text { ár. } a l+\text { lat. pistu })^{10}$.

En el campo derivativo, el árabe ha dejado el sufijo -í, para formar gentilicios (ceutí, marroqui, yemení...) u otros sustantivos o adjetivos (jabali, maravedí, muladí, baladí...). El término «alfonsí» aparece en el siglo XIII para referirse a lo relativo a Alfonso X. En el campo de las preposiciones, de origen árabe es hasta $(<$ ar. hatta) y sus variantes adta, ata, hata, fasta ${ }^{11}$. Lo son también las partículas marras, de balde, en balde. Con respecto a los indefinidos, la voces fulano (< ár. fulan «uno», «cualquiera») y mengano (< ár. man kana «el que sea») son también de procedencia árabe ${ }^{12}$. Las interjecciones hala, ojalá, guay y la antigua ya «Oh».

También ha dejado influencia notable en el español al nivel de los aspectos semánticos y fraseológicos de la influencia árabe que se interpretan como aquellas palabras y expresiones románicas en cuanto a origen y forma, pero parcial o totalmente arabizadas en cuanto a su significado. ${ }^{13}$ Así, infante «niño que no habla» pasó a significar «hijo de noble», «hijo de rey», apoyándose en el termino árabe walad «hijo», «niño», «heredero al trono». Parecido fenómeno se observa en bidalgo, fidalgo, bijodalgo; hijo se relacionó con el árabe ibn «descendiente de primer grado», «relacionado con». Casos como esos son casa («casa»/«ciudad»), plata («lámina»/《plata») ${ }^{14}$. Al lado de estas palabras las costumbres árabes dejaron una serie de expresiones romances calcadas del árabe: quede usted con Dios, gracias a Dios, que Dios te lo pague, a la paz de Dios, que vaya con Dios, etc.

\section{EL PLANTEAMIENTO DEL PROBLEMA DESDE EL PUNTO DE VISTA TRADUCTOLÓGICO}

Los préstamos lingüísticos que dejó la lengua árabe en la lengua castellana y principalmente aquellas palabras que han evolucionado semánticamente adoptando nuevos significados en el diccionario español pueden representar un problema serio que afecta al proceso de la traducción para aquellos que no tienen un buen dominio del léxico de la lengua española, sobre todo cuando se trata de una traducción hecha por estudiantes arabo parlantes a través de un texto español. Estas palabras las

\footnotetext{
10 Lillo, Elena, La invasión árabe, los árabes y el elemento árabe en español, p.6. 12-04-07, www.cervantesvirtual.com.

${ }^{11}$ Idem, p.7.

12 Lleal, C. La formación de las lenguas romances peninsulares, Barcelona, Barcanova, 1999, pp. 190194.

13 Lillo, Elena, La invasión árabe, los árabes y el elemento árabe en español, p.7, www.cervantesvirtual.com.

${ }^{14}$ Cano Aguilar, R. El español a través de los tiempos, 1999, p.54.
} 
podemos clasificar bajo lo que se llama en la ciencia de la traductología los falsos amigos.

Los errores que cometen mis alumnos cuando están ante un texto que incluye palabras españolas de origen árabe en la asignatura de traducción del español al árabe en el departamento de Español me hicieron pensar en plantear este problema desde el punto de vista de la traducción, ya que los errores se repetían en cada trimestre y con distintos grupos. Este trabajo de investigación se apoyó en los estudiantes de la asignatura Traducción del Español al Árabe de la Universidad de Jordania y el corpus que sirve de base a la investigación es de lenguaje escrito y está formado por un tipo de prueba que consiste en un ejercicio de traducción de algunas frases españolas que incluyen palabras de origen árabe que han evolucionado semánticamente.

En lo que sigue vamos a exponer el ejercicio que se les dio a los estudiantes de traducción y a continuación exponemos los fallos que cometieron los estudiantes y que se clasifican bajo el problema "Los Falsos Amigos".

Las frases españolas que incluyen préstamos lingüísticos árabes y que se dieron a los estudiantes de traducción son las siguientes:

1. El alcalde ${ }^{15}$ de Sevilla visitó la Universidad.

2. Juan es un calilo. ${ }^{16}$

3. El niño tomó su jarabe. ${ }^{17}$

4. El zaratán ${ }^{18}$ es una enfermedad muy grave.

5. A Juan le ascendieron a alférez: ${ }^{19}$

6. Mi padre es alguacil ${ }^{2} \mathrm{en}$ el ayuntamiento de Madrid.

7. En la casa del campo hay una alberca. ${ }^{21}$

8. He cazado un jabali. 22

9. Juan es un merquino ${ }^{23}$ y un baladí.

${ }^{15} \mathrm{El}$ origen árabe es al-qadi y significa el juez

${ }^{16} \mathrm{El}$ origen árabe es qalil y significa un poco. El adjetivo calilo es una palabra que está en desuso y se encuentra solamente en el lenguaje escrito

${ }^{17} \mathrm{El}$ origen árabe es sarab y significa zumo.

${ }^{18} \mathrm{El}$ origen árabe saratan y significa cáncer

${ }^{19} \mathrm{El}$ origen árabe es alfaris y significa el caballero.

${ }^{20} \mathrm{El}$ origen árabe es uažr y significa ministro.

${ }^{21}$ El origen árabe es birka y significa piscina.

${ }^{22} \mathrm{El}$ origen es yabali y significa montañoso.

${ }^{23} \mathrm{El}$ origen árabe es miskin y significa pobre hombre. 
Las frases anteriores se propusieron a dos grupos de estudiantes universitarios arabo-parlantes de tercer año. El primer grupo era de 33 estudiantes y el segundo de 19 estudiantes, y entre los 52 estudiantes 9 alumnos cayeron en la trampa de los falsos amigos y les dieron a las palabras españolas de origen árabe el significado del origen árabe por la influencia de la lengua materna sin darse cuenta de la evolución semántica que sufrieron estos elementos léxicos. En lo que sigue exponemos las versiones árabes erróneas que dieron los alumnos con sus traducciones al español:

Primera frase:

V.O ${ }^{24}$ : El alcalde de Sevilla visitó la universidad.

V.A : Zara qadi 'Tshbiliya al-yami'a

T.E : El juez de Sevilla visitó la universidad

V.A : Zara qa 'id 'Isbbiliya al-yami' a

T.E : El líder de Sevilla visitó la universidad.

Segunda frase:

V.O : Juan es un calilo

V.A : Juan rayulun Sagir.

T.E : Juan es un hombre pequeño.

V.A : Juan rayulun qasir.

T.E : Juan es bajo.

V.A : Juan rayulun faqir

T.E : Juan es un hombre pobre.

Tercera frase:

V.O: El niño tomó su jarabe.

V.A: Sariba al-tifl sarabahu.

T.E: El niño tomó su zumo.

Cuarta frase:

V.O: El zaratán es una enfermedad muy seria.

V.A: Al-saratan maradun hatir.

24 Abreviaturas utilizadas: V.O (versión original en español), V.A (versión árabe del estudiante), T.E (traducción al Español de la versión que hizo el estudiante). 
T.E: El cáncer es una enfermedad seria.

Quinta frase:

V.O: A Juan le ascendieron a alférez.

V.A: Sbaha Juan farisan.

T.E: Juan llegó a ser un jinete.

V.A: 'Arakabu Juan 'ala al-hisan.

T.E: A Juan le hicieron subir al caballo.

V.A: Tamma ta'yin Juan farisan.

T.E: A Juan le nombraron como un jinete en el ejército.

Sexta frase:

V.O: Mi padre es un alguacil en el tribunal de Madrid.

V.A: 'Abi wazirir fi mahkamat Madrid.

T.E: Mi padre es un ministro en el tribunal de Madrid.

V.A: 'Abi 'alladi ya' malu waziran, zara mahkamat Madrid.

T.E: Mi padre que trabaja como un ministro visitó el tribunal de Madrid.

Séptima frase:

V.O: En la casa del campo hay una alberca.

V.A: Fi al-bayt alladi fi almazra' a yuyad birkah.

T.E: En la casa del campo hay una piscina.

Octava frase:

V.O: He cazado un jabalí.

V.A: Stadtu hayawan yabali.

T.E: he cazado un animal montañoso.

Novena frase:

V.O: Juan es un mezquino.

V.A: Juan rayulun miskin.

T.E: Juan es un hombre pobre.

V.A: Juan rayulun tayyb. 
T.E: Juan es un hombre bueno.

\section{ANÁLISIS DE ERRORES}

La dificultad y la facilidad de la traducción están vinculadas de una forma directa con la cercanía y la lejanía de las dos lenguas entre las cuales se efectúa el proceso de la traducción, puesto que el efectuar una traducción entre dos lenguas cercanas entre sí etimológica, estructural y culturalmente representa menos obstáculos y tropiezos para el traductor, mientras que realizar traducciones entre dos lenguas alejadas entre sí etimológica, estructural y culturalmente como el árabe y el español entraña evidentes implicaciones para la práctica de la traducción y, por consiguiente, para el estudio y la enseñanza de la misma ${ }^{25}$.

El árabe y el español se clasifican en el grupo de las lenguas entre las que se dan diferencias considerables a todos los niveles, siendo las dos lenguas pertenecientes a dos bagajes lingüísticos y culturales muy alejados; pero pese a esas muchas diferencias tienen muchas cosas en común también, debido a una presencia árabe que duró ocho siglos en la península Ibérica.

Entre lo común que tienen las dos lenguas están las palabras españolas de origen árabe que se quedaron vivas en el uso y que entraron en el diccionario como palabras españolas reconocidas por los lingüistas.

Como se ha dicho anteriormente, estos préstamos lingüísticos pueden representar trampas y tropiezos para el aprendiz árabe desde el punto de vista traductológico, ya que cuando un alumno árabe está ante un texto que incluye palabras españolas de origen árabe tiende a darles a estas palabras los significados que tienen en su lengua materna sin tener en consideración la evolución semántica que sufrieron esas palabras, puesto que algunas de ellas adquirieron nuevos significados con el paso del tiempo. Ello se ve muy claramente en los fallos que cometieron nuestros alumnos de traducción en la traducción al árabe de algunas frases españolas que incluyen palabras de este tipo.

$\mathrm{Si}$ nos fijamos muy cuidadosamente en las traducciones árabes dadas a las oraciones españolas podemos deducir lo siguiente:

- Si el grado de semejanza en los sonidos es el mismo entre la palabra original y la palabra prestada, el alumno tiende a darle a esa palabra el significado existente en su lengua materna, descuidando la evolución semántica que sufrió, como figura en la séptima y la octava oraciones cuando le dio a la palabra alberca el significado de piscina y a la palabra jabalí el significado de montañoso, puesto que en árabe yabalí es el nombre relativo de la palabra yabal "montaña"; también como

${ }^{25}$ Peña, Salvador, Traductología, Universidad de Málaga/Manuales, 1981, p. 29. 
en la tercera frase, cuando se le dio a la palabra jarabe el significado de zumo.

- Pero si el grado de semejanza en la pronunciación no es igual, puesto que algunos préstamos se han adecuado a la pronunciación española, sufriendo un cambio en el orden de la colocación de las letras en la misma palabra o perdiendo una letra, el alumno tiende a darle a la palabra el significado de la palabra árabe más cercana en la pronunciación, como se ve muy obviamente en la primera oración cuando se le dieron a la palabra alcalde los significados de juez y líder, puesto que la pronunciación de las dos palabras originales en árabe que son al-qadi y al-qa'id es muy semejante a la palabra prestada en español.

Pero en otros casos el contexto lingǘ́stico hace que el alumno se confunda y le hace caer en correspondencias inadecuadas para dar la traducción correspondiente, ya que a veces el significado del original de la palabra prestada en la lengua materna del alumno no concuerda con el contexto en el que la palabra en cuestión va actualizada, y en este caso el alumno tiende a darle a la palabra un significado que le acepte la oración o incluso tiende a cambiar el significado entero de la oración para que vaya con el significado que tiene el origen de la palabra en su lengua materna, como en la sexta oración cuando un alumno en la segunda versión cambió el significado de la oración, y como también en la segunda y tercera traducciones de la quinta frase cuando el alumno cambió el significado de la frase española original.

La traducción de la segunda frase representa un caso interesante, ya que la palabra calilo cuyo origen árabe es qalil y que significa en español poco se utiliza en árabe como adjetivo o pronombre indefinido para referirse a una cantidad o a un número reducido ${ }^{26}$, igual que poco en español, aunque la palabra calilo actualmente significa tonto.

En las versiones que se hicieron de la oración Juan es un calilo, como se ve en las versiones siguientes, los alumnos tendieron a cambiar el significado de la oración haciendo entrar la palabra rayulun que significa hombre para que sus traducciones tengan un significado aceptable, puesto que si hubieran vertido la oración según el significado original de la palabra en su lengua materna, la oración no habría tenido un significado y se habría traducido como Juan es poco; por ello tendieron a cambiar el significado de qalil "poco" en otros como pequeño, bajo o pobre que son adjetivos aceptables para calificar al nombre propio Juan.

${ }^{26}$ Díaz, Regino, Larousse, diccionario general de la lengua española, Madrid, Larousse Editorial, 1997, p.968. 
V.O : Juan es un calilo

V.A : Juan rayulun sagir.

T.E : Juan es un hombre pequeño.

V.A : Juan rayulun qasir.

T.E : Juan es bajo.

V.A : Juan rayulun faqir

T.E : Juan es un hombre pobre.

Por lo que atañe a la novena frase y sus dos traducciones al árabe, la palabra mezquino, cuyo origen árabe es miskin, siempre tiene un significado positivo ${ }^{27} \mathrm{y}$ nunca un sentido peyorativo o negativo, como ocurre en el caso del español en el que la palabra mezquino puede tener los significados de avaro o tacaño ${ }^{28}$; por eso, como vemos en las oraciones, los alumnos le dieron a la palabra española los significados pobre hombre y hombre bueno, por la influencia del significado que tiene originalmente en su lengua materna:

V.O: Juan es un mezquino.

V.A: Juan rayulun miskin.

T.E: Juan es un pobre hombre.

V.A: Juan rayulun tayyb.

T.E: Juan es un hombre bueno.

El dominio del léxico representa una de las dificultades más relevantes en la adquisición de una lengua extranjera, con todo lo que ello conlleva de variedad y complejidad, tanto en sus relaciones sintagmáticas como pragmáticas ${ }^{29}$.

El 17\% de los alumnos sometidos a la prueba cayeron en la trampa de los falsos amigos por no tener un buen dominio del léxico español y creemos que aquellos alumnos al ver las palabras españolas prestadas del árabe sintieron confianza por tenerlas en su lengua materna y les dieron el significado del origen árabe sin recurrir al diccionario español, que es exigido en el aula de la clase de traducción. Esta interferencia de la lengua materna en el aprendiz disminuye si el alumno está viviendo en la sociedad lingǘstica en la que se habla español, pero no desaparece del todo. También en muchos casos, como vemos en los ejemplos de la prueba, el contexto

${ }^{27}$ En árabe significa una persona digna de compasión: pobre hombre.

${ }^{28}$ Idem, p.794.

${ }^{29}$ Fernández, Sonsoles, Interlengua y análisis de errores, Madrid, Edelsa, 1997, p.69. 
lingüístico no es capaz de eliminar todas las ambigüedades a las que el alumno debe enfrentarse. Por ello creemos que el uso del diccionario para los alumnos de traducción siempre es imprescindible cuando están ante una palabra cuyo significado puede representar ambigüedad o confusión.

Entre las 4300 palabras españolas prestadas del árabe existe un número considerable que han evolucionado semánticamente adquiriendo nuevos significados en el diccionario español. Siendo nuestro trabajo actual una investigación que se centra en esta clase de palabras, hemos sacado la lista siguiente después de una lectura cuidadosa de El diccionario de arabismos de Federico Corriente y del libro Léxico de las palabras de origen árabe de Mekinassi. Son palabras españolas de origen árabe que han cambiado de significado y que se pueden clasificar bajo los falsos amigos entre el árabe y el español. 


\begin{tabular}{|c|c|c|c|c|}
\hline $\begin{array}{c}\text { El significado del } \\
\text { origen árabe en } \\
\text { español }\end{array}$ & $\begin{array}{c}\text { El significado de la } \\
\text { palabra española en } \\
\text { español }\end{array}$ & $\begin{array}{l}\text { El origen } \\
\text { en árabe }\end{array}$ & $\begin{array}{c}\text { Palabra en } \\
\text { español }\end{array}$ & Letra \\
\hline El juez & $\begin{array}{l}\text { Presidente del } \\
\text { ayuntamiento de cada } \\
\text { pueblo o término } \\
\text { municipal }\end{array}$ & Al-qadi & Alcalde & A \\
\hline La rojez & $\begin{array}{c}\text { Tejido de lana con } \\
\text { varios dibujos y colores } \\
\text { con que se cubre el piso }\end{array}$ & Al-humra & Alfombra & \\
\hline El elefante & $\begin{array}{l}\text { Pieza grande del juego } \\
\text { de ajedrez }\end{array}$ & Al-fil & Alfil & \\
\hline Cubrecama/alfombra & $\begin{array}{l}\text { La piedra baja del } \\
\text { molino de aceite }\end{array}$ & Al-farš & Alfarje & \\
\hline Latido / vibración & Dolor de corazón & & Algafacán & \\
\hline El sabio & Médico & Al-Faqih & Alfaquí & \\
\hline El impuesto & $\begin{array}{l}\text { Cierta contribución que } \\
\text { pagaban los } \\
\text { musulmanes y judíos en } \\
\text { los reinos cristianos }\end{array}$ & Al-farda & Alfarda & \\
\hline El regalo & Soborno & Al-hadiyah & Alfadia & \\
\hline $\begin{array}{l}\text { El que aplica } \\
\text { ventosas }\end{array}$ & El barbero & Al-hayyamm & Alfajeme & \\
\hline La necesidad & Estimación, precio & Al-haya & Alfaya & \\
\hline La habitación & $\begin{array}{c}\text { Cámara alta para } \\
\text { recoger o conservar } \\
\text { granos }\end{array}$ & Al-gurfah & Algorfa & \\
\hline El ministro & $\begin{array}{c}\text { Oficial inferior de } \\
\text { justicia que ejecuta las } \\
\text { ordenes del tribunal a } \\
\text { quien sirve }\end{array}$ & Al-wazir & Alguacil & \\
\hline El cargador & Bestia de carga & Al-hammal & Alhamel & \\
\hline El hilo & Joya & $\mathrm{Al}$-jayt & Alhaite & \\
\hline El movimiento & $\begin{array}{l}\text { Demostración de afecto, } \\
\text { ira, queja o admiración. }\end{array}$ & Al-harakah & Alharaca & \\
\hline La necesidad & $\begin{array}{l}\text { Joya, adorno o mueble } \\
\text { precioso. cosa de valor }\end{array}$ & Al-haya & Alhaja & \\
\hline Ardiente/caliente & Adarce & Al-harraqah & Alhurreca & \\
\hline El cementerio & Cementerio musulmán & $\begin{array}{c}\text { Al- } \\
\text { maqbarah }\end{array}$ & Almacabara & \\
\hline El faro & Torre de las mezquitas & Al-manarah & Alminar & \\
\hline El que llama & $\begin{array}{l}\text { Venta publica de bienes } \\
\text { y muebles con licitación }\end{array}$ & Al-munadi & Almoneda & \\
\hline
\end{tabular}




\begin{tabular}{|c|c|c|c|c|}
\hline $\begin{array}{c}\text { El significado del } \\
\text { origen árabe en } \\
\text { español }\end{array}$ & $\begin{array}{c}\text { El significado de la } \\
\text { palabra española en } \\
\text { español }\end{array}$ & $\begin{array}{l}\text { El origen } \\
\text { en árabe }\end{array}$ & $\begin{array}{c}\text { Palabra en } \\
\text { español }\end{array}$ & Letra \\
\hline & y puja & & & \\
\hline Las rosas & $\begin{array}{l}\text { Flor del naranjo, del } \\
\text { limonero }\end{array}$ & Al-zahr & Azahar & \\
\hline El deseo & Huerto, granja & Al-munyah & Almunia & \\
\hline El riego & $\begin{array}{l}\text { Noria grande, ramal o } \\
\text { conducto de aguas }\end{array}$ & Al-siqayah & Azacaya & \\
\hline La columna & $\begin{array}{l}\text { Barra de hierro que } \\
\text { servía de cerrojo para } \\
\text { cerrar puertas y } \\
\text { ventanas }\end{array}$ & Al-'amud & Alamud & \\
\hline El armario & $\begin{array}{c}\text { Hueco hecho en la } \\
\text { pared, con puertas y } \\
\text { anaqueles para guardar } \\
\text { cosas. }\end{array}$ & AL-jazanah & Alacena & \\
\hline fiel & $\begin{array}{c}\text { Oficial que en el antiguo } \\
\text { contrastaba las pesas y } \\
\text { medidas }\end{array}$ & Al-'amin & Alamín & \\
\hline Donde se reza & Alfombra & Al-musalla & Almofalla & \\
\hline Parte interna, forro & Piel curtida de carnero & Bitana & Badana & B \\
\hline local & De clase inferior & Baladi & Baladí & \\
\hline poco & Tonto & Qalil & Calilo & $\mathrm{C}$ \\
\hline Electricidad & Ámbar & Kahruba' & Carabe & \\
\hline Infiel & Cruel, rústico & Kœfir & Cafre & \\
\hline lingote & Barra de hierro dulce & Sabikah & Cibica & \\
\hline Fabrica grande & $\begin{array}{l}\text { Parte resguardada } \\
\text { artificialmente, en aguas } \\
\text { navegables para las } \\
\text { embarcaciones }\end{array}$ & $\begin{array}{l}\text { Dar al- } \\
\text { sina'ah }\end{array}$ & Dársena & $\mathrm{D}$ \\
\hline $\begin{array}{l}\text { Registro, oficina del } \\
\text { estado }\end{array}$ & $\begin{array}{l}\text { Banco con brazos o sin } \\
\text { ellos por lo común sin } \\
\text { respaldo y con } \\
\text { almohadones sueltos }\end{array}$ & Diwan & Diván & \\
\hline Energía & $\begin{array}{l}\text { Cada una de las tongas } \\
\text { o hileras horizontales de } \\
\text { ladrillos que se forman } \\
\text { en el horno para } \\
\text { cocerlos }\end{array}$ & Taqah & Daga & \\
\hline Saliente, visible & $\begin{array}{l}\text { Impedir, retardar una } \\
\text { cosa }\end{array}$ & Bariz & Embarazar & $\mathrm{E}$ \\
\hline $\begin{array}{l}\text { El que acuña la } \\
\text { moneda }\end{array}$ & $\begin{array}{l}\text { Cada una de las casillas } \\
\text { cuadradas e iguales del } \\
\text { tablero de ajedrez }\end{array}$ & Al-sakkak & Escaque & \\
\hline
\end{tabular}




\begin{tabular}{|c|c|c|c|c|}
\hline $\begin{array}{c}\text { El significado del } \\
\text { origen árabe en } \\
\text { español }\end{array}$ & $\begin{array}{c}\text { El significado de la } \\
\text { palabra española en } \\
\text { español }\end{array}$ & $\begin{array}{l}\text { El origen } \\
\text { en árabe }\end{array}$ & $\begin{array}{c}\text { Palabra en } \\
\text { español }\end{array}$ & Letra \\
\hline La mitad de & $\begin{array}{l}\text { Defecto de una tabla o } \\
\text { madero que les impide } \\
\text { ser perfectamente lisos } \\
\text { o rectos }\end{array}$ & Filqah & Falca & $\mathrm{F}$ \\
\hline Contento & $\begin{array}{l}\text { Nombre con que se } \\
\text { distinguió en Marruecos } \\
\text { a cada uno de los } \\
\text { individuos de ciertas } \\
\text { familias españolas allí en } \\
\text { el siglo VIII, los cuales } \\
\text { siguieron siendo } \\
\text { cristianos, y al fin } \\
\text { volvieron y se } \\
\text { establecieron en castilla } \\
\text { en el año } 1390 .\end{array}$ & Farhan & Farrán & \\
\hline Tiempo libre & Haraganería, dejadez & Farag & Faranga & \\
\hline Uno entre dos & Bulto o lió de ropa & Farda & Farda & \\
\hline moneda & $\begin{array}{c}\text { Moneda de cobre de } \\
\text { poco valor }\end{array}$ & Fulus & Felús & \\
\hline Azada, hacha & Gorro de fieltro rojo & $\mathrm{Fa}{ }^{\prime} \mathrm{s}$ & Fez & \\
\hline Hotel & $\begin{array}{l}\text { Lugar de hospedaje y } \\
\text { almacén en Marruecos }\end{array}$ & Funduq & Fondac & \\
\hline Desgraciada & $\begin{array}{l}\text { Pereza, desidia o poca } \\
\text { gana de hacer una cosa }\end{array}$ & Galban & Galbana & G \\
\hline Botín & $\begin{array}{c}\text { Hurto pequeño y } \\
\text { frecuente }\end{array}$ & Ganimah & Galima & \\
\hline Multa & $\begin{array}{l}\text { Regalos que se hacen a } \\
\text { una familia con motivo } \\
\text { feliz }\end{array}$ & Garamah & Garama & \\
\hline Oeste & Viento sudoeste & Garbi & Garbino & \\
\hline Ignorante & Tonto & Yahil & Gilí & \\
\hline Cantidad & $\begin{array}{c}\text { Arma blanca que usan } \\
\text { los marroquíes }\end{array}$ & Kammiyah & Gumía & \\
\hline Triste & $\begin{array}{l}\text { Avaro, mezquino, } \\
\text { miserable }\end{array}$ & Hazin & Hacino & $\mathrm{H}$ \\
\hline Preservador & Guarda & Hafid & Hafiz & \\
\hline Movimiento & $\begin{array}{l}\text { Expedición militar de } \\
\text { tropas en Marruecos }\end{array}$ & Harakah & Harca & \\
\hline Emigración & $\begin{array}{c}\text { Era de los musulmanes } \\
\text { que comienza el jueves } \\
15 \text { de Julio del año } 622 \\
\text { de J.C. cuando el } \\
\text { profeta árabe }\end{array}$ & Hiyrah & Hégira & \\
\hline
\end{tabular}




\begin{tabular}{|c|c|c|c|c|}
\hline $\begin{array}{c}\text { E1 significado del } \\
\text { origen árabe en } \\
\text { español }\end{array}$ & $\begin{array}{c}\text { El significado de la } \\
\text { palabra española en } \\
\text { español }\end{array}$ & $\begin{array}{l}\text { El origen } \\
\text { en árabe }\end{array}$ & $\begin{array}{c}\text { Palabra en } \\
\text { español }\end{array}$ & Letra \\
\hline & $\begin{array}{l}\text { Muhammad huyó de la } \\
\text { Meca a Medina }\end{array}$ & & & \\
\hline Derecho & Alboroque & $\mathrm{Haq}$ & Hoque & \\
\hline Montañoso & Cerdo salvaje & Yabali & Jabalí & $\mathrm{J}$ \\
\hline Popular & $\begin{array}{l}\text { Dícese de una especie } \\
\text { de manzana Silvestre y } \\
\text { pequeña }\end{array}$ & $\check{S}_{\text {Sacbi }}{ }^{\prime}$ & Jabí & \\
\hline Fortalecida & $\begin{array}{l}\text { Madre de hilo .viga } \\
\text { maestro }\end{array}$ & Hasina & Jácena & \\
\hline Preparación & $\begin{array}{c}\text { Cualquier adorno que se } \\
\text { pone a las caballerías. } \\
\text { ropa o vestido }\end{array}$ & Yihaz & Jaez & \\
\hline El que teje & $\begin{array}{l}\text { Especie de almalafa } \\
\text { usada en el norte de } \\
\text { África, que sirve para } \\
\text { cubrirse las mujeres }\end{array}$ & $\mathrm{Ha}^{\prime} \mathrm{ik}$ & Jaique & \\
\hline Salida del sol & $\begin{array}{c}\text { Viento de levante. } \\
\text { Sudeste }\end{array}$ & Šuruq & Jaloque & \\
\hline Bonita & $\begin{array}{l}\text { Agua que corre de las } \\
\text { aceitunas apiladas }\end{array}$ & Yamilah & Jamila & \\
\hline Viejo & Valentón, perdonavidas & Šaij & Jaque & \\
\hline $\begin{array}{c}\text { Mitad. Dolor que } \\
\text { afecta la mitad de la } \\
\text { cabeza }\end{array}$ & $\begin{array}{l}\text { Dolor de cabeza más o } \\
\text { menos duradero }\end{array}$ & Šaqiqa & Jaqueca & \\
\hline Bebida/Zumo & $\begin{array}{l}\text { Cualquier bebida } \\
\text { excesivamente dulce }\end{array}$ & Šarab & Jarabe & \\
\hline Socio & $\begin{array}{c}\text { Reunir en un mismo caz } \\
\text { las hilas de agua de } \\
\text { varios propietarios para } \\
\text { regar }\end{array}$ & Šarik & Jaricar & \\
\hline Honorable, honesto & $\begin{array}{l}\text { Rozagante, vistoso, bien } \\
\text { compuesto o adornado }\end{array}$ & Šarif & Jarifo & \\
\hline Jirón & Tela gruesa y tosca & Jerqa & Jerga & \\
\hline Noche & $\begin{array}{l}\text { Fiesta o baile nocturno } \\
\text { entre los moriscos }\end{array}$ & Laylah & Leila & $\mathrm{L}$ \\
\hline Cámara privada & $\begin{array}{l}\text { Recinto reservado en } \\
\text { una mezquita, destinado } \\
\text { para el imán }\end{array}$ & Maqsurah & Macsura & M \\
\hline Cerrada & Compuerta & Muglaqah & Maglaca & \\
\hline $\begin{array}{l}\text { Referente a } \\
\text { Mohamed }\end{array}$ & $\begin{array}{c}\text { Que profesa la religión } \\
\text { islámica }\end{array}$ & Muhammadi & Mahometano & \\
\hline Pañuelo & Prenda de cuero o tela & Mandil & Mandil & \\
\hline
\end{tabular}




\begin{tabular}{|c|c|c|c|c|}
\hline $\begin{array}{c}\text { El significado del } \\
\text { origen árabe en } \\
\text { español }\end{array}$ & $\begin{array}{c}\text { El significado de la } \\
\text { palabra española en } \\
\text { español }\end{array}$ & $\begin{array}{l}\text { El origen } \\
\text { en árabe }\end{array}$ & $\begin{array}{c}\text { Palabra en } \\
\text { español }\end{array}$ & Letra \\
\hline cercado & $\begin{array}{c}\text { fuerte, colgada del } \\
\text { cuello, sirve en ciertos } \\
\text { oficios para proteger la } \\
\text { ropa. Delantal } \\
\text { Cédula que por lo } \\
\text { común se adhiere a las } \\
\text { piezas de la tela, cajas, } \\
\text { etc. }\end{array}$ & Marbat & Marbete & \\
\hline Hazmerreír & $\begin{array}{l}\text { Figura, a veces redícula } \\
\text {,hecha de cartón, tela o } \\
\text { alambre, con que una } \\
\text { persona puede taparse el } \\
\text { rostro para no ser } \\
\text { reconocida }\end{array}$ & Masjarah & Máscara & \\
\hline Silo subterráneo & Prisión subterránea & Matmurah & Mazmorra & \\
\hline Presumido/arrogante & $\begin{array}{c}\text { Hombre entremetido } \\
\text {,bullicioso y de poco } \\
\text { provecho }\end{array}$ & Mutaayref & Mequetrefe & \\
\hline Pobre & Avaro, tacaño & Miskin & Mezquino & \\
\hline Riesgo & $\begin{array}{l}\text { Venta fingida o } \\
\text { simulada que se hace, o } \\
\text { cuando se vente } \\
\text { teniendo prevenido } \\
\text { quién compre aquello } \\
\text { mismo a menos precio }\end{array}$ & Mujatara & Mohatra & \\
\hline gloriosa & $\begin{array}{l}\text { Monte alto con jarales y } \\
\text { maleza }\end{array}$ & Mayidah & Moheda & \\
\hline Estacionado & $\begin{array}{c}\text { Místico que profesa en } \\
\text { una ermita }\end{array}$ & Murabit & Morabito & \\
\hline Arabista & $\begin{array}{l}\text { Aplicase al cristiano que } \\
\text { vivió antiguamente } \\
\text { entre los árabes de la } \\
\text { España musulmana }\end{array}$ & Musta'rib & Mozárabe & \\
\hline $\begin{array}{c}\text { El juego } \\
\text { Separar, disuadir }\end{array}$ & $\begin{array}{c}\text { Baraja } \\
\text { Marcharse } \\
\text { precipitadamente }\end{array}$ & $\begin{array}{l}\text { Al-lacib } \\
\text { Naha }\end{array}$ & $\begin{array}{l}\text { Naipe } \\
\text { Naja }\end{array}$ & $\mathrm{N}$ \\
\hline Infusión & $\begin{array}{l}\text { Pequeño estanque o } \\
\text { pozuelo en que se } \\
\text { ponen a curtir las pieles } \\
\text { Parte alta de la cerviz, } \\
\text { correspondiente al lugar } \\
\text { en que se une el } \\
\text { espinazo con la cabeza }\end{array}$ & Naq'ah & Noque & \\
\hline
\end{tabular}




\begin{tabular}{|c|c|c|c|c|}
\hline $\begin{array}{c}\text { El significado del } \\
\text { origen árabe en } \\
\text { español }\end{array}$ & $\begin{array}{c}\text { El significado de la } \\
\text { palabra española en } \\
\text { español }\end{array}$ & $\begin{array}{l}\text { E1 origen } \\
\text { en árabe }\end{array}$ & $\begin{array}{l}\text { Palabra en } \\
\text { español }\end{array}$ & Letra \\
\hline Lo que tiene ventana & $\begin{array}{c}\text { Parpalla (moneda } \\
\text { antigua) }\end{array}$ & Abu-taqah & Pataca & $\mathrm{P}$ \\
\hline Recipiente de arcilla & $\begin{array}{l}\text { Bojito. Redoma de } \\
\text { vidrio muy usada en } \\
\text { algunas provincias para } \\
\text { beber vino a chorro }\end{array}$ & Barniyya & Porrón & \\
\hline Puesto & $\begin{array}{l}\text { Témplate o pabellón de } \\
\text { estilo oriental y } \\
\text { generalmente abierto } \\
\text { por todos lados, que se } \\
\text { construye en }\end{array}$ & Kušk & Quiosco & Q \\
\hline Monasterio sufí & $\begin{array}{c}\text { Azoteas ,jardines ,etc } \\
\text { Convento, ermita }\end{array}$ & Ribat & Rábida & $\mathrm{R}$ \\
\hline Retorno, repetición & $\begin{array}{c}\text { Periodo breve de } \\
\text { fortuna, más } \\
\text { comúnmente en el juego }\end{array}$ & Ray'ah & Racha & \\
\hline Silla de camello & $\begin{array}{c}\text { Granja, casa o predio en } \\
\text { el campo. }\end{array}$ & Rahil & Rafal & \\
\hline Espuma & $\begin{array}{l}\text { Remate superior de la } \\
\text { caña de azúcar }\end{array}$ & Raguah & Ragua & \\
\hline $\begin{array}{c}\text { Grano de arena, } \\
\text { suelo arenoso }\end{array}$ & $\begin{array}{l}\text { Lecho natural de las } \\
\text { aguas pluviales cuando } \\
\text { caen copiosamente }\end{array}$ & Ramlah & Rambla & \\
\hline Prado/jardín & Cementerio árabe & Rawdah & Rauda & \\
\hline Hogaza & Torta, hornazo & Ragif & Regaifa & \\
\hline Romano & $\begin{array}{l}\text { Cristiano entre los } \\
\text { árabes de España }\end{array}$ & Rumi & Romí & \\
\hline Al esté, oriental & $\begin{array}{l}\text { Natural de Arabia feliz u } \\
\text { oriundo de ella }\end{array}$ & Šarqi & Sarraceno & S \\
\hline Referente al sultán & $\begin{array}{l}\text { Moneda de oro fino } \\
\text { usada en el imperio } \\
\text { turco }\end{array}$ & Sultani & Soltaní & \\
\hline Plato & $\begin{array}{l}\text { Cestillo o canastillo } \\
\text { pequeño hecho de } \\
\text { mimbres }\end{array}$ & Tabaq & Tabaque & $\mathrm{T}$ \\
\hline Ventana & Alacena pequeña & Taqah & Taca & \\
\hline Obediencia & Comarca, distrito & Ta'ah & Taha & \\
\hline Exitoso, ganador & Jugador & Dafur & Tahúr & \\
\hline Secta & $\begin{array}{c}\text { Bandería, parcialidad. } \\
\text { los reyes de taifa en la } \\
\text { España árabe }\end{array}$ & Ta'ifah & Taifa & \\
\hline Bordador & Sastre & Tarazi & Tarasi & \\
\hline Presa & Embarcación usada & Tarida & Tarida & \\
\hline
\end{tabular}

I.S.S.N. $1132-0265$ 


\begin{tabular}{|c|c|c|c|c|}
\hline $\begin{array}{c}\text { E1 significado del } \\
\text { origen árabe en } \\
\text { español }\end{array}$ & $\begin{array}{c}\text { El significado de la } \\
\text { palabra española en } \\
\text { español }\end{array}$ & $\begin{array}{l}\text { E1 origen } \\
\text { en árabe }\end{array}$ & $\begin{array}{c}\text { Palabra en } \\
\text { español }\end{array}$ & Letra \\
\hline Cuadruplicación & $\begin{array}{l}\text { desde el siglo XII en el } \\
\text { mediterráneo } \\
\text { Sala grande }\end{array}$ & Tarbi ${ }^{6}$ & Tarbea & \\
\hline Científicos & $\begin{array}{c}\text { Doctor en las ciencias } \\
\text { islámica }\end{array}$ & 'ulama' & Ulema & $\mathrm{U}$ \\
\hline Mancha & $\begin{array}{l}\text { Parte de tierra baja, llena } \\
\text { y fértil }\end{array}$ & Buq'ah & Vega & V \\
\hline Vestido, ropa & $\begin{array}{l}\text { Vestidura que } \\
\text { antiguamente se ponía } \\
\text { debajo de la armadura }\end{array}$ & Malbas & Velmez & \\
\hline Ministro & $\begin{array}{l}\text { Ministro en un estado } \\
\text { árabe, primer ministro }\end{array}$ & Wazir & Visir & \\
\hline $\begin{array}{c}\text { Tanque, } \\
\text { naturalmente para } \\
\text { agua }\end{array}$ & $\begin{array}{l}\text { Cantarera o sitio donde } \\
\text { se ponen cántaros }\end{array}$ & Sahriy & Zafariche & Z \\
\hline Roca & Escombra & Sajrah & Zafra & \\
\hline Seco & Tosco, inculto, grosero & Yaf & Zafio & \\
\hline Rincón, ángulo & $\begin{array}{l}\text { En Marruecos especie } \\
\text { de ermita, mezquita con } \\
\text { la tumba de un santo }\end{array}$ & Zawiyah & Zagaüía & \\
\hline $\begin{array}{l}\text { El dueño de la } \\
\text { ciudad }\end{array}$ & $\begin{array}{l}\text { Magistrado que había en } \\
\text { el antiguo Aragón con } \\
\text { jurisdicción civil y } \\
\text { criminal }\end{array}$ & $\begin{array}{l}\text { Sahibu al- } \\
\text { madina }\end{array}$ & Zalmedina & \\
\hline Cáncer & $\begin{array}{l}\text { Cáncer de los pechos en } \\
\text { la mujer }\end{array}$ & Saratan & Zaratán & \\
\hline Presión/callejuela & $\begin{array}{c}\text { Excavación larga y } \\
\text { angosta que se hace en } \\
\text { la tierra }\end{array}$ & Zanqah & Zanja & \\
\hline Color azul & Ojos de color azul claro & 'Zraq & Zarco & \\
\hline
\end{tabular}




\section{REFERENCIAS}

CABO PAN, J. L.: El legado del árabe. Consejería de Educación y Ciencia en Bélgica. $<$ http://www.sgci.mec.es/be/media/pdfs/articulos/Mosaico083.pdf>

CANO AGUILAR, R. (1999): El español a través de los tiempos, Madrid, Arco/libros.

CORRIENTE, F. (1977): Agramatical sketch of the spanish Arabia of bundle, Madrid, Instituto hispano-árabe de cultura.

CORRIENTE, F. (1999): Diccionario de arabismos y voces afines en iberorromance, Madrid, Gredos.

CORTÉS, J. (1996): Diccionario de árabe culto moderno, Madrid, Gredos.

DÍAZ, R. \& ALONSO, M. (1997): Diccionario general de la lengua española, Madrid, Larousse Bordas.

FARGAL, M. \& SHUNNAQ, A. (1999): Translation with reference to English and Arabic, Irbid, Dar al-Hilal.

FERNÁNDEZ, S. (1997): Interlengua y análisis de errores en el aprendizaje del español como lengua extranjera, Madrid, Edelsa.

GARCÍA YEBRA, V. (1989): En torno a la traducción: teoría, crítica e historia, Gredos, Madrid.

LEECH, G. (1974): Semantics, London, Harmondsworth.

LLEAL, C. (1999): La formación de las lenguas romances peninsulares, Barcelona, Barcanova.

MAÍlLO SALGADO, F. (1998): Los arabismos del castellano en la Baja Edad Media: consideraciones históricas y filológicas, Salamanca, universidad de Salamanca.

MEKINASSI, A. (1965): Léxico de las palabras españolas de origen árabe, Tetuán, Cremades.

MENÉNDEZ PIDAL, R.: Manual de Gramática Histórica Española, Madrid, Ed. Espasa Calpe, 1973.

MUÑIZ CANCHÓN, C. (2001): “Alcance de la gramática en la traducción. Los falsos amigos gramaticales". Revista española de lingüística, 31(2001).

NAYYAR, J. (1986): Traité de traducción, Beirut, Dar al-Masiq.

NEWMARK, P. (1999): Manual de traducción, Madrid, Cátedra.

MOUNIN, G. (1976): Linguistique et traduction, Bruxelles.

PEÑA, S. \& GUERRO, M. (1981): Traductología, Universidad de Málaga, Málaga.

REDA, Y. M. (1995): Al-Mu'in al-Wasit, Líbano, Librairie du Liban.

SÁNCHEZ ALBORNOZ, C.: La España musulmana, Madrid, Espasa Calpe, 1984.

STEWART, D.: Arabic influence on Spanish language, Literature, and culture. Emory Resourses on the Middle East.

$<$ http://www.mesas.emory.edu/gmesc/pdf/9_Spain_Unit_Part_1.pdf > 
TORO LILLO, E.: "La invasión árabe. Los árabes y el elemento árabe en español”. Instituto Cervantes.

<http://www.cervantesvirtual.com/servlet/SirveObras/013505319666822861906 80/p0000001.htm\#I_0>

WIKIPEDIA: http://es.wikipedia.org/wiki/Falsos_amigos. 